\title{
Surface tension and capillary waves at the nematic-isotropic interface in ternary mixtures of liquid crystal, colloids, and impurities
}

\author{
V. Popa-Nita ${ }^{a}$ \\ Faculty of Physics, University of Bucharest, P.O. Box MG-11, Bucharest 077125, Romania \\ P. Oswald \\ Laboratoire de Physique de l'Ecole Normale Supérieure de Lyon, 46 Allée d'Italie, 69364 Lyon Cedex 07, \\ France
}

(Received 14 May 2007; accepted 21 July 2007; published online 12 September 2007)

In mixtures of thermotropic liquid crystals with spherical poly(methyl methacrylate) particles, self-supporting networklike structures are formed during slow cooling past the isotropic-to-nematic phase transition. Experimental results support the hypothesis that a third component, alkane remnants slowly liberated from the particles, plays a crucial role. A theoretical model, based on the phenomenological Landau-de Gennes, Carnahan-Starling, and hard-sphere crystal theories, is developed to describe the continuous phase separation in a ternary nematic-impurity-colloid mixture. The interfacial tension and the dispersion relation of the surface modes of the nematic-isotropic interface are determined. The colloids decrease the interfacial tension and the damping rate of surface waves, whereas impurities act in an opposite way. This should strongly influence the formation of abovementioned networklike structures and could help explain some of their rheological properties. (C) 2007 American Institute of Physics. [DOI: 10.1063/1.2772251]

\section{INTRODUCTION}

Recently, there has been a considerable interest in colloidal particles dispersed in anisotropic media, principally liquid crystals. ${ }^{1-6}$ When particles are immersed in a nematic liquid crystal, the director field is distorted by the particles, generating interesting new phenomena. Depending on the type of anchoring (planar or homeotropic) and on its penetration length $L_{p}=K / W$ (with $K$ the Frank elastic constant and $W$ the anchoring energy of the nematic at the particle surface $^{7,8}$ ), different structures develop around a single particle. For instance, for homeotropic anchoring a point defect ("hedgehog") or a "Saturn ring" can nucleate nearby the particle depending on whether $L_{p}$ is small (strong anchoring) or large (weak anchoring) with respect to $R$ (for a review about this problem, see Ref. 6). These defects give rise to highly anisotropic, long-range elastic interactions of dipolar or quadrupolar type between the dispersed particles.

These induced interactions lead to original patterns, such as chaining which is the most commonly observed at low concentration of particles. $^{9-11}$

The situation becomes different at large concentration of particles because the latter tends to separate from the nematic phase to form an isotropic phase (liquid or solid) which coexists with the nematic phase. This separation is due (only in part, as we shall see later) to the distortions of the director field cost energy. ${ }^{12-17}$ It turns out that the first studies have focused on dispersions of $\approx 200 \mathrm{~nm}$ size poly(methyl methacrylate) (PMMA) spheres in 4'-pentyl-4-cyanobiphenyl (5CB) or $N$-4-methoxybenzylidene-4'-butylaniline. Microscopic observations have shown that below the nematic-to-

\footnotetext{
${ }^{a)}$ Author to whom correspondence should be addressed. Electronic mail: v.popanita@gmail.com
}

isotropic phase transition temperature, the two phases coexist by forming a three-dimensional network which gives to the mixture the consistency of a soft cellular solid. More precisely, the particles are densely packed in relatively thin walls constituting the isotropic phase, while the cavities in between are filled with almost pure nematic liquid crystal.

In these experiments, the network was formed in the weak-anchoring limit as the particle radius was very small $\left(R \ll L_{p}\right)$. This condition is nevertheless not crucial because similar networks were recently observed in the opposite strong-anchoring limit by using micrometer-sized particles for which $R \gg L_{p} .{ }^{16-18}$ These studies showed, in addition, that experiments (including those reported before in Refs. 12-15) were not dealing with binary but with ternary systems. The reason is that during the process of particle preparation, the remnant alkane cannot be removed completely. As a consequence, it slowly diffuses into the suspension after homogenization. This phenomenon may be important, firstly, because the particles are wetted by a layer of alkane which can change the anchoring of the liquid crystal molecules on their surfaces. Secondly, the nematic-to-isotropic transition temperature is lowered while a biphasic region shows up in the presence of alkane, favoring the formation of the cellular structure. It is this second point that we propose to study theoretically.

More precisely, we shall first develop a model based on the Landau-de Gennes mean field treatment to determine the phase diagram of a ternary system consisting of a nematic liquid crystal, impurities (a non-nematogenic fluid), and colloids. We will see that in this mixture, a biphasic region between the isotropic and the nematic phases appears below the nematic-to-isotropic transition temperature of the pure liquid crystal. We shall then study the static and dynamic 
properties of the nematic-isotropic interface (more precisely, its surface tension and its capillary waves). As we have already treated this problem in the particular case of a binary mixture of liquid crystal and nonmesogenic impurities (see the previous article ${ }^{19}$ ), we shall mainly focus our attention on the influence of added colloids on the properties of the nematic-isotropic interface.

The paper is organized as follows. In Sec. II, we introduce the free energy of a nonuniform ternary liquid crystalimpurity-colloid mixture. In Sec. III, we present the binary (without colloids) and ternary phase diagrams. In Sec. IV, a general expression for the nematic-isotropic surface tension is derived. This quantity is then calculated explicitly for typical values of the parameters. Its large variations as a function of the colloid and the impurity concentrations are emphasized and are discussed in the framework of the experiments reported before. In Sec. V, we give the dynamical governing equations necessary to calculate the dispersion relation for capillary waves at the nematic-isotropic interface. Numerical results about the damping rate of the waves are then presented and discussed. Finally, in Sec. VI, we draw some conclusions and directions for future work.

\section{FREE ENERGY}

The mixture is characterized by the volume fractions of the three components,

$$
\Phi_{i}=\frac{N_{i} v_{i}}{\sum_{i=1}^{3} N_{i} v_{i}} \quad \text { with } \sum_{i=1}^{3} \Phi_{i}=1,
$$

where $N_{i}$ is the number of molecules of component $i$ ( $i=1$ defines the liquid crystal, $i=2$ the non-nematogenic fluid, and $i=3$ the colloids) and $v_{i}$ is the volume of a particle of component $i$. In what follows, we consider that the volumes of a nematic and impurity molecule are equal $\left(v_{1}=v_{2}=v\right)$ and the volume of a colloid is $v_{R}=(4 \pi / 3) R^{3}$, where $R$ is the radius of a colloid particle (with $v_{R} \gg v$ ). The orientational order of the mixture is characterized by the nematic order parameter $Q_{\alpha \beta}$ (with Cartesian indices $\alpha, \beta=1,2,3$ ) which, in the case of uniaxial nematic state, can be expressed as ${ }^{7}$

$$
Q_{\alpha \beta}=S\left(3 n_{\alpha} n_{\beta}-\delta_{\alpha \beta}\right) / 2 .
$$

The unit vector $\mathbf{n}$ is the nematic director which fixes the average local uniaxial orientation of the liquid crystal molecules. Due to the head-to-tail invariance of molecules, the cases $\pm \mathbf{n}$ refer to the same state. The scalar $S$ is the orientational order parameter. The isotropic liquid is characterized by $S=0$ and a perfectly oriented nematic phase (i.e., with no fluctuations about $\mathbf{n}$ ) would correspond to $S=1$. In this paper, we suppose to simplify that $\mathbf{n}$ is fixed in space and time, so that the relevant physics is only governed by the scalar order parameter $S(\mathbf{r}, t)$. We note that this is an idealization which is neither true in general during the relaxation nor close to the interface even at equilibrium. However, previous studies ${ }^{20}$ suggest that the slowest relaxation modes approximately fulfill this condition when the director anchoring is homeotropic at the interface.
The free energy functional of the system is given by

$$
\begin{aligned}
F\left[\Phi_{1}, \Phi_{2}, \Phi_{3}, Q_{\alpha \beta}\right]= & \int\left[f\left(\Phi_{1}, \Phi_{2}, \Phi_{3}, Q_{\alpha \beta}\right)\right. \\
& +\frac{1}{2} K_{\Phi}\left(\nabla \Phi_{2}\right)^{2}+\frac{1}{2} L_{1}\left(\partial_{\gamma} Q_{\alpha \beta}\right)^{2} \\
& \left.+\frac{1}{2} L_{2}\left(\partial_{\alpha} Q_{\alpha \beta}\right)^{2}\right] d V,
\end{aligned}
$$

where $K_{\Phi}$ is a phenomenological coefficient. The elastic constants $L_{1}$ and $L_{2}$ are related to the Frank-Oseen elastic constants by the relations $K_{1}=K_{3}=9 S_{n}^{2}\left(L_{1}+L_{2} / 2\right) / 2$ and $K_{2}$ $=9 S_{n}^{2} L_{1} / 2$, where $S_{n}$ is the bulk nematic order parameter. In the so-called "one-constant approximation" $\left(K_{1}=K_{2}=K_{3}\right.$ $\left.=9 S_{n}^{2} K_{S} / 2\right), L_{1}=K_{S}$ and $L_{2}=0$, values which we consider in this paper. We neglect contributions from a gradient in the colloid concentration. The reason is that the contribution of colloids to the total interfacial tension is negligibly small due to the large size of the particles (indeed, it scales as the thermal energy divided by the size of the particle squared). Since the colloids are at least ten and more likely 100 times larger than the molecules of liquid crystal, their contribution is insignificant. The interfacial tension between phaseseparated colloidal phases can be as low as a few millionth of a $\mathrm{N} / \mathrm{m} .^{21}$ We have also neglected the coupling term $K_{0}\left(\partial_{\alpha} \Phi_{2}\right)\left(\partial_{\beta} Q_{\alpha \beta}\right)$, since previous study indicates that its influence to interfacial tension is small. ${ }^{19}$

The bulk free energy density consists of three parts,

$$
\begin{aligned}
f\left(\Phi_{1}, \Phi_{2}, \Phi_{3}, Q_{\alpha \beta}\right)= & f_{\text {mix }}\left(\Phi_{1}, \Phi_{2}\right)+f_{\text {coll }}\left(\Phi_{3}\right) \\
& +f_{\mathrm{lc}}\left(\Phi_{1}, \Phi_{2}, \Phi_{3}, Q_{\alpha \beta}\right) .
\end{aligned}
$$

The first term is the free energy density of the isotropic mixing of liquid crystal and non-nematic fluid and according to the Flory theory is given by ${ }^{22}$

$$
f_{\text {mix }}=\frac{k_{B} T}{v}\left(\Phi_{1} \ln \Phi_{1}+\Phi_{2} \ln \Phi_{2}+\chi \Phi_{1} \Phi_{2}\right),
$$

where $k_{B}$ is the Boltzmann constant, $T$ the absolute temperature, and $\chi=\chi_{12}$ the dimensionless Flory-Huggins interaction parameter which characterizes the isotropic interaction energy (divided by $k_{B} T$ ) between the liquid crystal and impurity molecules. ${ }^{22}$ In this paper, we consider $\chi$ as a constant. Because $v_{R} \gg v$, the interaction between the colloidal particles and the liquid crystal and impurity molecules can be neglected $\left[\chi_{13}=\chi_{23}=0\right.$ as these two quantities are of the order of $\left.\left(v / v_{R}\right)^{1 / 3} \chi \ll \chi\right]$.

The second term in Eq. (4) is the free energy density of a colloidal suspension, 


$$
f_{\text {coll }}=\frac{k_{B} T}{v_{R}}\left\{\begin{array}{ccc}
\Phi_{3} \ln \Phi_{3}+\Phi_{3}^{2}\left(4-3 \Phi_{3}\right) /\left(1-\Phi_{3}\right)^{2}, & \Phi_{3}<0.52, & \text { liquid } \\
u_{0} \Phi_{3}+3 \Phi_{3} \ln \Phi_{3} /\left(1-\Phi_{3} / \Phi_{c}\right), & \Phi_{3}>0.52, & \text { solid, }
\end{array}\right.
$$

where the two expressions are matched by the adjustable parameter $u_{0}=1.793$ at the concentration of 0.52 , while $\Phi_{c}$ $=0.637$ is the random close-packing fraction. The first line in Eq. (6) is the Carnahan-Starling excess free energy density of a hard-sphere suspension, ${ }^{23}$ while the second line is the free energy density of a hard-sphere crystal based on free volume considerations. $^{24}$

The third term in Eq. (4) is the Landau-de Gennes free energy density, ${ }^{7}$ which describes the weakly first-order nematic-to-isotropic phase transition,

$$
\begin{aligned}
f_{\mathrm{lc}}= & \Phi_{1}\left[a\left(T-\left(1-\lambda_{2} \Phi_{2}-\lambda_{3} \Phi_{3}\right) T^{*}\right) Q_{\alpha \beta} Q_{\beta \alpha}\right. \\
& \left.-B Q_{\alpha \beta} Q_{\beta \gamma} Q_{\gamma \alpha}+C\left(Q_{\alpha \beta} Q_{\beta \alpha}\right)^{2}\right] .
\end{aligned}
$$

In this expression, $T^{*}$ is the spinodal temperature of the isotropic phase of the pure liquid crystal, and $a, B$, and $C$ are three constant coefficients. For 5CB for instance, they have the following values: $a \approx 3.5 \times 10^{4} \mathrm{~J} / \mathrm{m}^{3} \mathrm{~K}, \quad B \approx 7.1$ $\times 10^{5} \mathrm{~J} / \mathrm{m}^{3}$, and $C \approx 4.3 \times 10^{5} \mathrm{~J} / \mathrm{m}^{3}$. ${ }^{3,25}$ We shall take these values in the following. The term $\left(1-\lambda_{2} \Phi_{2}-\lambda_{3} \Phi_{3}\right) T^{*}$ ensures that the nematic-to-isotropic transition temperature decreases linearly as a function of the impurity and colloid concentrations. The physical origin of the coupling parameters $\lambda_{2}$ and $\lambda_{3}$ is discussed in Refs. 19 and 14, respectively. In this paper, we consider them as phenomenological parameters.

For the pure liquid crystal $\left(\Phi_{1}=1\right.$ and $\left.\Phi_{2}=\Phi_{3}=0\right)$, using the form of Eq. (2) for $Q_{\alpha \beta}$, the bulk free energy density has the well-known expression,

$$
f_{\mathrm{lc}}=\frac{3}{2} a\left(T-T^{*}\right) S^{2}-\frac{3}{4} B S^{3}+\frac{9}{4} C S^{4} .
$$

This equation describes a weakly first-order nematic-toisotropic phase transition. At $T=T_{\mathrm{NI}}=T^{*}+B^{2} / 24 a C$, the two phases, nematic $\left(S_{\text {nem } 0}=B / 6 C\right)$ and isotropic $\left(S_{\text {iso }}=0\right)$, coexist in equilibrium.

\section{PHASE DIAGRAMS}

\section{A. Basic equations for calculating phase diagrams}

The calculation of the static phase diagrams only requires to know the bulk free energy density. For convenience, we introduce nondimensional quantities. The temperature is replaced by the reduced temperature $\tau$ $=\left(T-T^{*}\right) /\left(T_{\mathrm{NI}}-T^{*}\right)$. The orientational order parameter is normalized with respect to its value in the pure liquid crystal at the transition temperature $T_{\mathrm{NI}}: \bar{S}=S / S_{\text {nem } 0}$. Setting $\bar{f}_{\text {coll }}$ $=f_{\text {coll }} /\left(k_{B} T / v_{R}\right)$ and $\bar{\lambda}_{2,3}=24 a T^{*} C \lambda_{2,3} / B^{2}$, the dimensionless free energy $\bar{f}=f / f_{0}$ (with $f_{0}=B^{4} / 24^{2} C^{3}$ ) reads after omitting the bar notation,

$$
\begin{aligned}
f= & \Gamma(1+\delta \tau)\left(\Phi_{1} \ln \Phi_{1}+\Phi_{2} \ln \Phi_{2}+\chi \Phi_{1} \Phi_{2}\right) \\
& +\Gamma_{R}(1+\delta \tau) f_{\text {coll }}+\Phi_{1}\left[\left(\tau+\lambda_{2} \Phi_{2}+\lambda_{3} \Phi_{3}\right) S^{2}-2 S^{3}+S^{4}\right],
\end{aligned}
$$

where $\Gamma=k_{B} T^{*} /\left(v f_{0}\right), \Gamma_{R}=k_{B} T^{*} /\left(v_{R} f_{0}\right)$, and $\delta=\left(T_{\mathrm{NI}}-T^{*}\right) / T^{*}$.

Below $T_{\mathrm{NI}}$, a phase separation may occur depending on the initial composition of the mixture. During this process, the system of average composition $\left(\Phi_{2}, \Phi_{3}\right)$ splits into a nematic phase of composition $\left(\Phi_{2 \text { nem }}, \Phi_{3 \text { nem }}\right)$ and an isotropic phase of composition $\left(\Phi_{2 \text { iso }}, \Phi_{3 \text { iso }}\right)$. The values of $\Phi_{2 \text { nem }}$, $\Phi_{3 \text { nem }}, \Phi_{2 \text { iso }}$, and $\Phi_{3 \text { iso }}$ are given at equilibrium by the equations (remembering that $\Phi_{1}=1-\Phi_{2}-\Phi_{3}$ in each phase)

$$
\begin{aligned}
& \mu_{2} \equiv \mu_{2}\left(S_{\text {nem }}, \Phi_{2 \text { nem }}, \Phi_{3 \text { nem }}\right)=\mu_{2}\left(0, \Phi_{2 \text { iso }}, \Phi_{3 \text { iso }}\right), \\
& \mu_{3} \equiv \mu_{3}\left(S_{\text {nem }}, \Phi_{2 \text { nem }}, \Phi_{3 \text { nem }}\right)=\mu_{3}\left(0, \Phi_{2 \text { iso }}, \Phi_{3 \text { iso }}\right), \\
& g \equiv g\left(S_{\text {nem }}, \Phi_{2 \text { nem }}, \Phi_{3 \text { nem }}\right)=g\left(0, \Phi_{2 \text { iso }}, \Phi_{3 \text { iso }}\right),
\end{aligned}
$$

where $\mu_{2}\left(S, \Phi_{2}, \Phi_{3}\right), \mu_{3}\left(S, \Phi_{2}, \Phi_{3}\right)$, and $g\left(S, \Phi_{2}, \Phi_{3}\right)$ are defined by the equations

$$
\begin{aligned}
& \mu_{2}\left(S, \Phi_{2}, \Phi_{3}\right)=\frac{\partial f}{\partial \Phi_{2}} ; \quad \mu_{3}\left(S, \Phi_{2}, \Phi_{3}\right)=\frac{\partial f}{\partial \Phi_{3}} \\
& g\left(S, \Phi_{2}, \Phi_{3}\right)=f-\mu_{2} \Phi_{2}-\mu_{3} \Phi_{3} .
\end{aligned}
$$

We emphasize that in the definition of grand potential $g\left(S, \Phi_{2}, \Phi_{3}\right), \mu_{2}$ and $\mu_{3}$ are the constant chemical potentials of the impurities and of the colloids, respectively. As for the orientational order parameter $S_{\text {nem }}$, it is obtained by minimizing the free energy density [Eq. (9)] with respect to $S$, which gives

$$
S_{\mathrm{nem}}=\frac{1}{4}\left(3+\sqrt{9-8\left(\lambda_{2} \Phi_{2}+\lambda_{3} \Phi_{3}+\tau\right)},\right.
$$

while $S_{\text {iso }}=0$. Note that, with our new notations, $S_{\text {nem } 0}=1$ in the pure liquid crystal $\left(\Phi_{2}=\Phi_{3}=0\right)$ at $\tau=1$. In the following, we discuss separately the cases of the binary and ternary diagrams.

\section{B. Binary phase diagram of the liquid crystal-impurity mixture}

This case corresponds to $\Phi_{3}=0$. To simplify, we denote by $\Phi$ the average impurity volume fraction $\Phi_{2}$ and by $1-\Phi$ the liquid crystal volume fraction $\Phi_{1}$. To compute the phase diagram, we must choose a value for $\Gamma$. This parameter depends on $f_{0}$, the value of the energy barrier to overcome at the nematic-to-isotropic phase transition in the pure liquid crystal. From typical values of $a, B$, and $C$ given above for $5 \mathrm{CB}$, we calculate $f_{0} \approx 5500 \mathrm{~J} / \mathrm{m}^{3}$. Volume $v$ represents in an ideal mixture the molecular volume. In the case of liquid crystal $5 \mathrm{CB}$, each molecule has the shape of a cylinder of length of $3 \mathrm{~nm}$ and of diameter of $0.5 \mathrm{~nm}$. That 


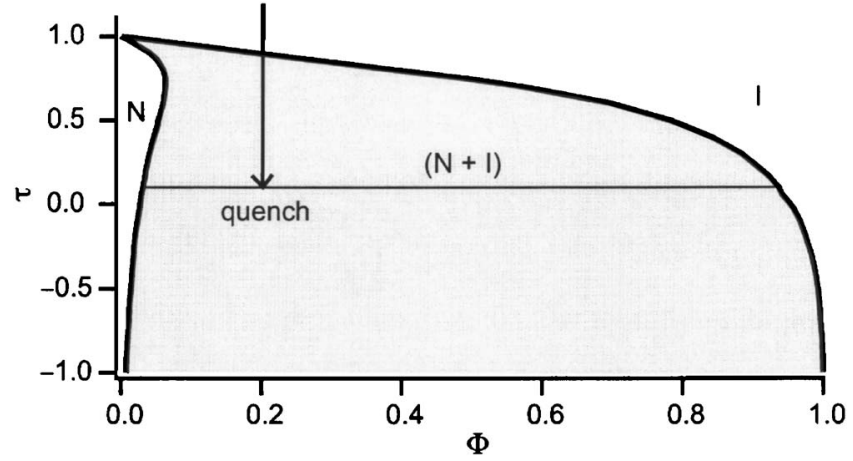

FIG. 1. Phase diagram for nematic-non-nematic binary mixture for $\Gamma=0.8$, $\lambda_{2}=1$, and $\chi=1$.

gives $v \approx 22.5 \times 10^{-27} \mathrm{~m}^{3}$. From this value, we calculate $\Gamma$ $\approx 300$. It turns out that if we perform the calculations with this value of $\Gamma$, we do not find any phase separation at the nematic-to-isotropic phase transition.

On the other hand, choosing a smaller value of $\Gamma$ allows us to find a phase diagram compatible with observations. ${ }^{26}$ Such a phase diagram, calculated for $\Gamma=0.8, \lambda_{2}=1$, and $\chi$ $=1$, is shown in Fig. 1. Consequently, we must decrease the value of $\Gamma$ by more than two orders of magnitude to obtain an acceptable phase diagram. That means that the mixing entropy of the liquid crystal and impurity molecules is smaller than expected. This is possible if molecules of each species form clusters. So $v$ must be considered as an effective volume (larger than the molecular volume) characterizing the nonideality of the mixture. In the following, we shall retain the phenomenological value $\Gamma=0.8$.

We now briefly describe the obtained phase diagram. For a temperature below $T_{\mathrm{NI}}\left(\tau_{\mathrm{NI}}=1\right)$, there exists a two-phase coexistence region between an isotropic (high- $\Phi$ ) and a nematic (low- $\Phi)$ phase. On decreasing temperature (note that the spinodal temperature of the isotropic phase of the pure liquid crystal corresponds to $\tau^{*}=0$ ), the biphasic region broadens. When the system is thermally quenched from the stable isotropic phase into the biphasic region, fluctuations of the concentration and of the orientational order occur, and nematic droplets nucleate. At the end of the process, the system separates into two phases of compositions $\Phi_{\text {nem }}$ and $\Phi_{\text {iso. }}$. For the quench represented by the arrow in Fig. 1, the initial average composition $\Phi=0.2$ at high temperature $\tau>1$ [corresponding to $\left.\Phi_{2} / \Phi_{1}=\Phi /(1-\Phi)=0.25\right]$ splits into $\Phi_{\text {nem }}=0.031$ and $\Phi_{\text {iso }}=0.935$ at temperature $\tau=0.1$. We note that in this example, the impurity is strongly rejected into the isotropic phase (as expected for nonmesogenic molecules).

In the next section, we establish the phase diagram for the ternary mixture at the reduced temperature $\tau=0.1$. We also discuss what happens when colloids or impurities are progressively added to the mixture.

\section{Ternary phase diagram of the liquid crystal-impurity-colloid mixture}

For the three-component system consisting of liquid crystal, impurities, and colloids, the equilibrium equations (10) involve four independent concentration variables, namely, two for each phase $\left(\Phi_{2 \text { nem }}\right.$ and $\Phi_{3 \text { nem }}$ for the nematic

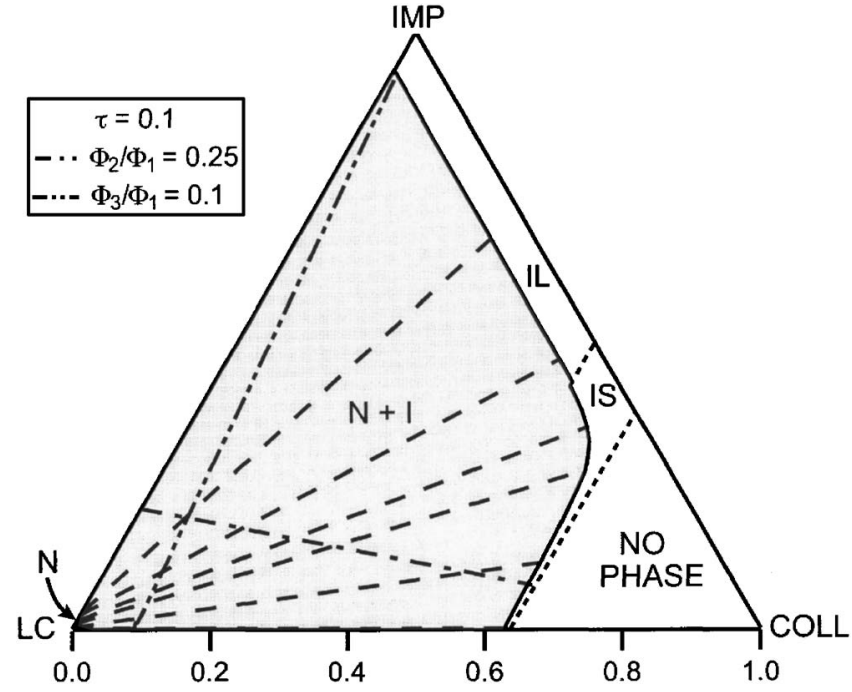

FIG. 2. Phase diagram calculated for a three-component system consisting of liquid crystal, impurities, and colloids. The solid curve refers to the binodal. Inside the gray region, there is phase separation. The dashed lines give the volume fractions of each species in the two phases at their intersections with the bimodal. Along the dashed-dotted line (the dotted line), $\Phi_{2} / \Phi_{1}$ $=0.25\left(\Phi_{3} / \Phi_{1}=0.10\right)$. In the isotropic phase, the hard-sphere colloid is in the liquid state (IL) below the coexistence boundary $\Phi_{3 \text { iso }}<0.52$, whereas it is in the isotropic solid state (IS) at higher concentration $0.52<\Phi_{3 \text { iso }}<\Phi_{\mathrm{c}}$ $=0.637$, where $\Phi_{c}$ is the random close-packing fraction.

phase, and $\Phi_{2 \text { iso }}$ and $\Phi_{3 \text { iso }}$ for the isotropic liquid). If one of these variables is specified, the other three are fixed by the three equations (10). It is possible, therefore, to compute at each reduced temperature $\tau$ the binodal curve which gives the two-phase equilibrium compositions of the threecomponent system.

An example of phase diagram is plotted in Fig. 2 for $\Gamma$ $=0.8, \lambda_{2}=1, \chi=1, \tau=0.1, \Gamma_{R}=0.006$, and $\lambda_{3}=1.35$. The last two values have been chosen in order that there exists a two-phase coexistence region (gray region in the phase diagram) between a rich-in-colloid isotropic phase and a nematic phase in which the colloid concentration practically cancels. This is typically what is observed experimentally with colloidal particles of diameter in the range of a few tens of nanometers. We recall that the colloids are expelled from the nematic phase because they distort the director field, which is unfavorable energetically. We shall note that points representing the nematic phase are located in the lower part of the left side of the triangle (corresponding to $\Phi_{3} \approx 0$ ). We shall also emphasize that in the isotropic phase, the hard-sphere colloid is in the liquid state below the coexistence boundary $\Phi_{3 \text { iso }}<0.52$ and in the solid state at higher concentration $0.52<\Phi_{3 \text { iso }}<\Phi_{\mathrm{c}}=0.637$, where $\Phi_{\mathrm{c}}$ is the random closepacking fraction. Finally, for $\Phi_{3 \text { iso }}>0.637$, no phase is defined by the model.

From this phase diagram, we can describe two complementary situations which could be test experimentally. First, we can add impurities to a given mixture of liquid crystal and colloids of constant ratio $\Phi_{3} / \Phi_{1}$, for instance $\Phi_{3} / \Phi_{1}$ $=0.10$. This is what happens in the experiments performed with PMMA colloids where the remnant alkane slowly diffuses in the mixture or is directly added. ${ }^{12,14,15}$ The followed path in this case corresponds to the dashed-double dotted 
line in the ternary phase diagram. Starting from the bottom of phase diagram, we immediately see that adding impurities to the mixture tends to simultaneously increase the impurity concentration and decrease the colloid concentration in the isotropic phase, whereas the liquid crystal remains almost pure in the nematic phase. As a result, the colloid, which initially forms a solid phase, melts to give a disordered suspension when the impurity concentration is sufficiently large (in this example, when $\Phi_{2}>0.09$ ).

Second, we can add colloids to a well-defined mixture of liquid crystal and impurities (imposing, for instance, $\left.\Phi_{2} / \Phi_{1}=0.25\right)$. In this case, we follow the dashed-dotted line in the ternary phase diagram. Starting from the left of the phase diagram, we immediately note that when $\Phi_{3}$ increases, the impurity concentration strongly decreases in the isotropic liquid, whereas the liquid crystal remains almost pure in the nematic phase as in the previous case. As for the colloidal particles, they first form a disordered suspension in the isotropic liquid to finally solidify when $\Phi_{3}$ exceeds 0.52 .

In the next section, we show that changing the concentrations of the different species along a given path leads to large variations of the nematic-isotropic surface tension.

\section{INTERFACIAL TENSION}

We consider a planar nematic-isotropic interface of unit surface area in equilibrium and take the $z$ axis perpendicular to the interface. We choose the origin $z=0$ such that the nematic phase $\left(S=S_{\text {nem }}\right)$ lies in the region $z<0$ and the isotropic phase $(S=0)$ in the region $z>0$. The free energy functional (3) can be expressed as (by using real variables)

$$
\begin{aligned}
F= & \int_{-\infty}^{\infty} d z\left[f+\frac{1}{2} K_{\Phi_{2}}\left(d_{z} \Phi_{2}\right)^{2}+\frac{1}{2} K_{\Phi_{3}}\left(d_{z} \Phi_{3}\right)^{2}\right. \\
& \left.+\frac{1}{2} K_{S}\left(d_{z} S\right)^{2}\right] .
\end{aligned}
$$

The interfacial tension $\gamma$ is defined as the difference, per unit area of the interface, between the actual free energy of the system and that of the two phases if each were uniform and isolated. It is thus given by

$$
\begin{aligned}
\gamma= & f_{0} \int_{-\infty}^{\infty} d z\left[\Delta g+\frac{1}{2} l_{\Phi_{2}}^{2}\left(d_{z} \Phi_{2}\right)^{2}+\frac{1}{2} l_{\Phi_{3}}^{2}\left(d_{z} \Phi_{3}\right)^{2}\right. \\
& \left.+\frac{1}{2} l_{S}^{2}\left(d_{z} S\right)^{2}\right]
\end{aligned}
$$

where $\Delta g=g\left(S(z), \Phi_{2}(z), \Phi_{3 \text { nem }}\right)-g\left(0, \Phi_{2 \text { iso }}, \Phi_{3 \text { iso }}\right)$ is the dimensionless energy difference calculated from Eq. (11) and $S$ the order parameter normalized to its value in the pure liquid crystal $\left(S \equiv S / S_{\text {nem } 0}\right)$. The three correlation lengths are defined to be $l_{S}=\left(K_{S} S_{\text {nem } 0}^{2} / f_{0}\right)^{1 / 2}, l_{\Phi_{2}}=\left(K_{\Phi_{2}} / f_{0}\right)^{1 / 2}$, and $l_{\Phi_{3}}$ $=\left(K_{\Phi_{3}} / f_{0}\right)^{1 / 2}$. As we shall see later, they give the typical widths of the profiles in $S, \Phi_{2}$, and $\Phi_{3}$, respectively.

Minimizing the functional in Eq. (14) with respect to $\Phi_{2}(z), \Phi_{3}(z)$, and $S(z)$, we obtain the corresponding EulerLagrange equations for the equilibrium profiles of the order parameter and of the impurity and colloid compositions,

$$
\begin{aligned}
& l_{\Phi_{2}}^{2} d_{z}^{2} \Phi_{2}=\frac{\partial \Delta g}{\partial \Phi_{2}}, \\
& l_{\Phi_{3}}^{3} d_{z}^{2} \Phi_{3}=\frac{\partial \Delta g}{\partial \Phi_{3}}, \\
& l_{S}^{2} d_{z}^{2} S=\frac{\partial \Delta g}{\partial S},
\end{aligned}
$$

with the following boundary conditions $(i=2,3)$ :

$$
\left(\Phi_{i}, S\right)= \begin{cases}\left(\Phi_{\text {inem }}, S_{\text {nem }}\right) & \text { as } z \rightarrow-\infty \\ \left(\Phi_{\text {iso }}, 0\right) & \text { as } z \rightarrow \infty,\end{cases}
$$

and $d_{z} \Phi_{i}( \pm \infty)=d_{z} S( \pm \infty)=0$.

Multiplying Eq. (15) by $d_{z} \Phi_{2}$, Eq. (16) by $d_{z} \Phi_{3}$, and Eq. (17) by $d_{z} S$, adding the resulting equations, and then integrating once with respect to $z$, we obtain

$$
\Delta g=\frac{1}{2} l_{\Phi_{2}}^{2}\left(d_{z} \Phi_{2}\right)^{2}+\frac{1}{2} l_{\Phi_{3}}^{2}\left(d_{z} \Phi_{3}\right)^{2}+\frac{1}{2} l_{S}^{2}\left(d_{z} S\right)^{2} .
$$

Using this expression to eliminate $\Delta g$ term from Eq. (14), the interfacial tension becomes

$$
\gamma=f_{0} \int_{-\infty}^{\infty}\left[l_{\Phi_{2}}^{2}\left(d_{z} \Phi_{2}\right)^{2}+l_{\Phi_{3}}^{2}\left(d_{z} \Phi_{3}\right)^{2}+l_{S}^{2}\left(d_{z} S\right)^{2}\right] d z .
$$

To calculate $\gamma$ we need the values of $K_{S}$ and $K_{\Phi_{i}}(i$ $=2,3)$. For the former, we use the experimental value of $K_{S}=2.1 \times 10^{-12} \mathrm{~N}$ given for 5CB in Ref. 25, from which we calculate $l_{S}=1.7 \times 10^{-8} \mathrm{~m}$ by taking the previous values of $S_{\text {nem } 0}$ and $f_{0}$. The stiffness coefficient $K_{\Phi_{2}}$ is unknown experimentally for a $5 \mathrm{CB}$-alkane mixture. For this reason, we propose to take $K_{\Phi_{2}} / K_{S}=40$, as in Ref. 27, which gives explicitly $K_{\Phi_{2}}=3.8 \times 10^{-10} \mathrm{~N}$ and $l_{\Phi_{2}}=2.6 \times 10^{-7} \mathrm{~m}$. As for $K_{\Phi_{3}}$, it must be very small in comparison with $K_{S}$ and $K_{\Phi_{2}}$ because of the "large" size of the particles, so we shall take $K_{\Phi_{3}}=0$. In the following, we set $K_{\Phi_{2}} \equiv K_{\Phi}\left(l_{\Phi_{2}} \equiv l_{\Phi}\right)$.

With this particular choice, the length scales are well separated because $l_{\Phi} \gg l_{S}$. A direct consequence is that the differential equations [(15)-(17)] are decoupled since on the distance over which each variable changes significantly, the others may be considered as constant. In practice, that means that in the right-hand side of each equation, Eqs. (15)-(17), the partial derivative can be replaced by a total derivative. As a result the typical distances over which $S$ and $\Phi$ change are given, respectively, by $l_{S}$ and $l_{\tilde{\phi}}$. More precisely, it can be checked numerically that the real profiles are very close to hyperbolic tangent profiles of the forms

$$
\begin{aligned}
& \Phi_{2}(z)=\frac{1}{2}\left(\Phi_{2 \text { nem }}+\Phi_{2 \text { iso }}+\left(\Phi_{2 \text { iso }}-\Phi_{2 \text { nem }}\right) \tanh \frac{z}{\sqrt{2} l_{\Phi}}\right), \\
& S(z)=\frac{S_{\text {nem }}}{2}\left(1-\tanh \frac{z}{\sqrt{2} l_{S}}\right) .
\end{aligned}
$$

From these profiles and Eq. (20), we can immediately calculate the interfacial tension, 

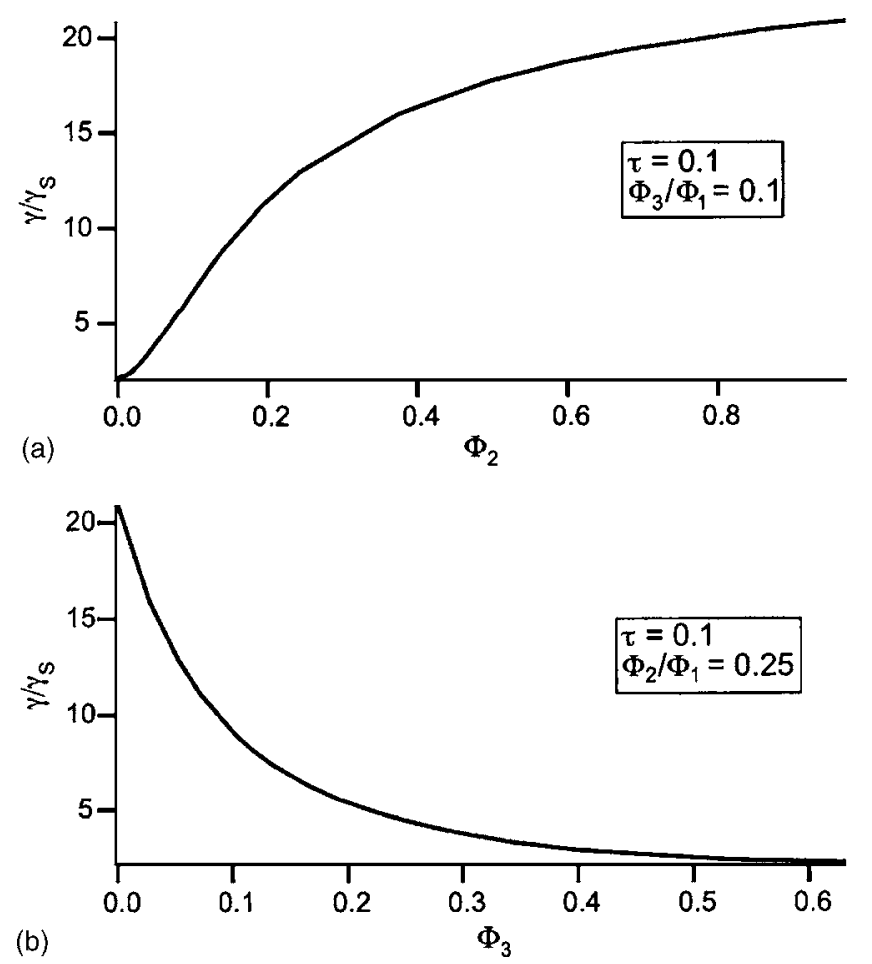

FIG. 3. (a) Nematic-isotropic reduced interfacial tension $\gamma / \gamma_{S}$ as a function of impurity volume fraction $\Phi_{2}$ for $\tau=0.1$ and $\Phi_{3} / \Phi_{1}=0.1$. (b) The same quantity as a function of colloid volume fraction $\Phi_{3}$ for $\tau=0.1$ and $\Phi_{2} / \Phi_{1}$ $=0.25$. $\gamma_{S}$ is the nematic-isotropic interfacial tension for the pure liquid crystal.

$$
\gamma / \gamma_{S}=S_{\mathrm{nem}}^{2}+\left(\Phi_{2 \text { iso }}-\Phi_{2 \mathrm{nem}}\right)^{2}\left(\frac{l_{\Phi}}{l_{S}}\right)
$$

where $\gamma_{S}$ is the surface tension of the pure liquid crystal: $\gamma_{S}=(\sqrt{2} / 6) f_{0} l_{S}$ [knowing that in this limiting case, the $S(z)$ profile given in Eq. (21) is exact]. Note that in Eq. (22), $S_{\text {nem }}$ is calculated from Eq. (12), while $\Phi_{2 \text { iso }}$ and $\Phi_{2 \text { nem }}$ are the concentrations calculated from the ternary phase diagram. We emphasize that all these quantities depend on the colloid concentration $\Phi_{3}$. As a consequence, the surface tension must change in the presence of the colloids, which is not obvious at the first sight from Eq. (22).

To illustrate this important point, let us return to the two experimental situations described at the end of the previous section. More precisely, let us discuss how the surface tension changes when impurities (colloids) are added to a colloid-liquid crystal mixture of a given composition $\Phi_{3} / \Phi_{1}$ (to an impurity-liquid crystal mixture of a given composition $\left.\Phi_{2} / \Phi_{1}\right)$. All calculations were performed at reduced temperature $\tau=0.1$, by taking as reference surface tension $\gamma_{S}$ of value of $2.2 \times 10^{-5} \mathrm{~N} / \mathrm{m}$.

In the first case (corresponding to the dashed-double dotted line of equation $\Phi_{3} / \Phi_{1}=0.10$ in Fig. 2), the reduced surface tension $\gamma / \gamma_{S}$ strongly increases when $\Phi_{2}$ increases [Fig. 3(a)]. In the second case (corresponding to the dasheddotted line of equation $\Phi_{2} / \Phi_{1}=0.25$ in Fig. 2), $\gamma / \gamma_{S}$ strongly decreases when $\Phi_{3}$ increases [Fig. 3(b)]. These large variations can be easily explained by considering that the term proportional to $S_{\text {nem }}^{2}$ in Eq. (22) is practically constant, whereas the second term, proportional to $\left(\Phi_{2 \text { iso }}-\Phi_{2 \text { nem }}\right)^{2}$ (al- ways very close to $\Phi_{2 \text { iso }}^{2}$ as the nonmesogenic impurities are expelled from the nematic phase) strongly increases (strongly decreases) when one adds impurities (colloids) to the mixture. This can be seen immediately from the phase diagram by moving along the dashed-double dotted line (the dashed-dotted line).

We can expect that these large variations of the surface tension play an important role in experiments. Indeed, our calculations show that adding a small amount of impurity can strongly increase the nematic-isotropic surface tension [Fig. 3(a)]. In contrast, adding colloids to an impure liquid crystal leads to an opposite effect [Fig. 3(b)]. This second effect could explain qualitatively why the mesh size of the observed cellular superstructure decreases when the concentration of colloids is increased. ${ }^{14}$ The elastic modulus $G^{\prime}$ should also depend on the impurity concentration. Such effects were indeed reported recently in experiments ${ }^{18}$ but they are not yet well documented.

Another question that arises is how to measure the surface tension. This could be performed by using the sessile (or pendant) drop method, ${ }^{8,28}$ provided that the colloid can flow in order that the drop equilibrates in the gravity field. According to our calculations, this condition is fulfilled for small concentrations of colloids or large concentrations of impurities. In practice, it will also be necessary to break the cellular structure which spontaneously forms during the phase transition in order to separate the nematic phase from the isotropic one. This could be done by centrifugation, the colloid being generally more dense than the nematic phase, but one must be careful to not separate the colloids from the rest of the mixture, which is an experimental challenge. An alternative method to estimate the surface tension would be to look at the damping rate of the capillary waves at the flat nematic-isotropic interface, for instance, by using new developed microscopy techniques which are more local than usual scattering techniques. This question, which represents in itself another experimental challenge, is analyzed in detail in the next section from a theoretical point of view.

\section{CAPILLARY WAVES}

\section{A. Equations of motion}

We assume that the heat diffusion is sufficiently rapid in order that the system remains at thermal equilibrium. We therefore ignore the equation for energy conservation and assume an isothermal system at a specified temperature. We further assume that the fluid is incompressible. Within these approximations, the equations of motion for the velocity and the nematic order parameter become ${ }^{29-31}$

$$
\begin{aligned}
& \partial_{\alpha} v_{\alpha}=0, \\
& \rho \frac{d v_{\alpha}}{d t}=\partial_{\beta}\left(-p \delta_{\alpha \beta}+\sigma_{\alpha \beta}^{d}+\sigma_{\alpha \beta}^{v}\right)+\frac{\delta F}{\delta \Phi_{2}} \partial_{\beta} \Phi_{2} \delta_{\alpha \beta}, \\
& 0=h_{\alpha \beta}+h_{\alpha \beta}^{v}-\lambda \delta_{\alpha \beta}-\epsilon_{\alpha \beta \gamma} \lambda_{\gamma},
\end{aligned}
$$

where $\rho$ is the density and $p$ the pressure, while $\lambda$ and $\lambda_{\gamma}$ are the Lagrange multipliers associated with conditions $\operatorname{Tr} Q=0$ and $Q_{\alpha \beta}=Q_{\beta \alpha}$, respectively. In this expression, $\alpha, \beta$, and $\gamma$ 
run from 1 to 3, summation over repeated indices is implied, $\epsilon_{\alpha \beta \gamma}$ is the Levi-Civita symbol, and $d / d t$ is the total time derivative $\partial / \partial t+\mathbf{v} \cdot \nabla$. The distortion stress $\sigma^{d}$ [which results from molecular displacement keeping the orientation fixed: $\mathbf{r} \rightarrow \mathbf{r}+\mathbf{u}(\mathbf{r})$ and $\left.Q_{\alpha \beta}(\mathbf{r}) \rightarrow Q_{\alpha \beta}^{\prime}\left(\mathbf{r}^{\prime}\right)=Q_{\alpha \beta}(\mathbf{r})\right]$ and the elastic molecular field $h$ [which results directly from the virtual orientational distortion: $\left.Q_{\alpha \beta}(\mathbf{r}) \rightarrow Q_{\alpha \beta}^{\prime}(\mathbf{r})\right]$ are obtained in the standard manner and read

$$
\begin{aligned}
& \sigma_{\alpha \beta}^{d}=-\frac{\partial F}{\partial\left(\partial_{\alpha} Q_{\gamma \rho}\right)} \partial_{\beta} Q_{\gamma \rho}, \\
& h_{\alpha \beta}=-\delta F / \delta Q_{\alpha \beta} .
\end{aligned}
$$

The viscous stress tensor $\sigma^{v}$ and the viscous molecular field $h^{v}$ are introduced through the consideration of entropy production in a dissipative flowing nematic. Within a tensorial description of the Ericksen-Leslie theory, ${ }^{31-33}$ they are given by

$$
\begin{aligned}
\sigma_{\alpha \beta}^{v}= & \beta_{1} Q_{\alpha \beta} Q_{\mu \nu} A_{\mu \nu}+\beta_{4} A_{\alpha \beta}+\beta_{5} Q_{\alpha \mu} A_{\mu \beta}+\beta_{6} Q_{\beta \mu} A_{\mu \alpha} \\
& +\frac{1}{2} \mu_{2} N_{\alpha \beta}-\mu_{1} Q_{\alpha \mu} N_{\mu \beta}+\mu_{1} Q_{\beta \mu} N_{\mu \alpha} \\
-h_{\alpha \beta}^{v} & =-\frac{1}{2} \mu_{2} A_{\alpha \beta}+\mu_{1} N_{\alpha \beta},
\end{aligned}
$$

where

$$
N_{\alpha \beta}=\frac{d Q_{\alpha \beta}}{d t}+Q_{\alpha \mu} W_{\mu \beta}-W_{\alpha \mu} Q_{\mu \beta}
$$

is the time rate of change of the order parameter with respect to the background fluid angular velocity, also known as the corotational time derivative. The quantities $\beta_{1}, \beta_{4}, \beta_{5}, \beta_{6}$, $\mu_{1}$, and $\mu_{2}=\beta_{6}-\beta_{5}$ are viscous coefficients which can be expressed in terms of the Leslie coefficients $\left(\alpha_{i}\right)$ and the value of the order parameter $S,{ }^{31}$ while $A_{\alpha \beta}=\frac{1}{2}\left(\partial_{\alpha} v_{\beta}+\partial_{\beta} v_{\alpha}\right)$ and $W_{\alpha \beta}=\frac{1}{2}\left(\partial_{\alpha} v_{\beta}-\partial_{\beta} v_{\alpha}\right)$ are, respectively, the symmetric and antisymmetric parts of the velocity gradient tensor.

The impurity composition equation of motion takes the Cahn-Hilliard form ${ }^{34}$

$$
\frac{d \Phi_{2}}{d t}=\Gamma_{\Phi} \nabla^{2}\left(\frac{\delta F}{\delta \Phi_{2}}\right),
$$

where the transport coefficient $\Gamma_{\Phi}$ is assumed to be constant. The complete dynamics is described by Eqs. (23)-(25) and (31).

We consider a two-dimensional flow with horizontal and vertical velocity components $u$ and $w$ in the $x$ and $z$ directions, respectively, and we simplify the expressions (28) and (29) by assuming that

$$
\beta_{1}=\beta_{5}=\beta_{6}=0, \quad \beta_{4}=2 \beta,
$$

which give $\mu_{2}=0 .{ }^{31}$ In terms of Leslie coefficients $\alpha_{i}$, these relations are equivalent to

$$
\begin{aligned}
& \alpha_{1}=\alpha_{5}=\alpha_{6}=0, \quad-\alpha_{2}=\alpha_{3}=\alpha=9 S^{2} \mu_{1} / 4, \\
& \alpha_{4}=\beta_{4}=2 \beta .
\end{aligned}
$$

Within these approximations, the coefficient $\beta$ describes the dissipation due to shear flow (shear viscosity), while $\mu_{1}$ is associated with the standard rotational viscosity $\gamma_{1}=\alpha_{3}-\alpha_{2}$ $=2 \alpha=9 S^{2} \mu_{1} / 2$.

Using these hypotheses, the basic Eqs. (23)-(25) and (31) take the following forms:

$$
\begin{aligned}
& 0=\partial_{x} u+\partial_{z} w, \\
& \rho \frac{d u}{d t}=-\partial_{x} p+\eta \nabla^{2} u-K_{S} \nabla^{2} S \partial_{x} S \\
& +\left(\frac{\partial f}{\partial \Phi_{2}}-K_{\Phi} \nabla^{2} \Phi_{2}\right) \partial_{x} \Phi_{2}, \\
& \rho \frac{d w}{d t}=-\partial_{z} p+\eta \nabla^{2} w-K_{S} \nabla^{2} S \partial_{x} S \\
& +\left(\frac{\partial f}{\partial \Phi_{2}}-K_{\Phi} \nabla^{2} \Phi_{2}\right) \partial_{z} \Phi_{2}, \\
& \mu_{1} \frac{d S}{d t}=-\frac{\partial f}{\partial S}+K_{S} \nabla^{2} S, \\
& \frac{d \Phi_{2}}{d t}=\Gamma_{\Phi} \nabla^{2}\left(\frac{\partial f}{\partial \Phi_{2}}-K_{\Phi} \nabla^{2} \Phi_{2}\right),
\end{aligned}
$$

where $\eta=\eta_{\text {iso }}=\beta$ in the isotropic phase (when $\Phi_{3}=0$ ), while $\eta=\eta_{\text {nem }}=(\alpha+2 \beta) / 2$ in the nematic phase [note that this viscosity corresponds to the second Miesowicz viscosity $\eta_{b}$ (Ref. 8) measured when the nematic phase is sheared parallel to the director].

In what follows, we shall suppose that the stationary planar nematic-isotropic interface (i.e., the base state of the system) is situated at $z=0$, such that the nematic lies in the region $z<0$ and the isotropic phase in the region $z>0$. The $x$ axis is taken in the direction of the wave vector $\mathbf{k}$ of the perturbation along the interface. This is possible without loss of generality if the system is isotropic in $x$ and $y$ directions, which implicitly assumes that the director anchoring on the interface is homeotropic and the biaxiality of the nematic phase is negligible. In this way, the wave number $k$ represents the modulus of the two-dimensional wave vector in the plane of the interface. Due to thermal fluctuations, small amplitude monochromatic waves of the form $\propto \exp (i k x-\Omega t)$ develop at the interface. In our notation, $k$ is the wave vector (real number) and $\Omega$ is the angular frequency. The latter quantity is generally a complex number whose the real part gives the relaxation time $1 / \mathcal{R}(\Omega)$ of the wave and the imaginary part, the phase velocity $\mathcal{I}(\Omega) / k$.

\section{B. Dispersion relation}

To obtain the dispersion relation we use the method of matched asymptotic expansions. ${ }^{35}$ The results obtained for similar systems using this method were extensively presented previously. ${ }^{19,36-38}$ The method consists of matching the solution obtained in an outer region, where $z$ is of the order of $l_{\eta}=\eta^{2} / \rho \gamma \simeq 1 \times 10^{-2} \mathrm{~m}$, to that calculated in an inner region in which $z$ is of the order of $l_{S}=\left(K_{S} S_{\text {nem } 0}^{2} / f_{0}\right)^{1 / 2}$ $\simeq 1.7 \times 10^{-8} \mathrm{~m}$. There are two outer regions (one, deep into the nematic phase, $z \rightarrow-\infty$, and the other deep into the iso- 
tropic phase, $z \rightarrow+\infty)$ in which the dominant physics is hydrodynamics, i.e., the dissipation due to the shear flow. In contrast, the variations of the nonconserved order parameter $S$ and of the conserved parameter $\Phi_{2}$ are the dominant processes in the inner region. ${ }^{19}$

The corresponding dispersion relation is given by ${ }^{38}$

$$
\Omega\left(\Omega-\Omega_{S \Phi}\right)=\Omega_{\eta}^{2} .
$$

The angular frequency $\Omega_{S \Phi}$ (corresponding to the inner region of size $l_{S}$ ) fixes the angular frequency in the limit $k$ $\rightarrow \infty$. It expresses in the form ${ }^{19}$

$$
\Omega_{S \Phi}=\frac{\gamma k^{2}}{\mu_{1} \gamma_{S} / K_{S}+\left(\Phi_{2 \text { iso }}-\Phi_{2 \mathrm{nem}}\right)^{2} / 2 k \Gamma_{\Phi}} .
$$

The difference $\left(\Phi_{2 \text { iso }}-\Phi_{2 \text { nem }}\right)$ is calculated for a colloid composition $\Phi_{3}$ different from 0 accordingly with the ternary phase diagram shown in Fig. 2.

As for $\Omega_{\eta}$, it gives the angular frequency in the opposite limit $k \rightarrow 0$ (corresponding to the outer region of dimension $\left.l_{\eta}\right)$. It is identical to the classical capillary-wave dispersion relation for a sharp interface. It reads ${ }^{39,40}$

$$
\Omega_{\eta}^{2}=\left[1+\frac{k\left(k_{\mathrm{nem}}^{2}+k_{\text {iso }}^{2}\right)-2 k^{3}}{\left(k_{\mathrm{nem}}+k_{\text {iso }}\right)\left(k^{2}-k_{\mathrm{nem}} k_{\mathrm{iso}}\right)}\right] \Omega_{0}^{2},
$$

where $\Omega_{0}^{2}=-\gamma k^{3} / 2 \rho$ is the capillary-wave dispersion relation for ideal (inviscid) fluids, $k_{\text {nem }}=k\left(1-\rho \Omega_{\eta} / \eta_{\text {nem }} k^{2}\right)^{1 / 2}$, and $k_{\text {iso }}=k\left(1-\rho \Omega_{\eta} / \eta_{\text {iso }} k^{2}\right)^{1 / 2}$. The latter expression is exact if the viscosities of the two phases are constant. It turns out that in our case, $\eta_{\text {iso }}$ corresponds to the viscosity of a disordered colloidal hard-sphere dispersion. As a consequence $\eta_{\text {iso }}$ is not constant but depends on the angular frequency given here by $\mathcal{R}(\Omega)$. According to de Kruif et al., ${ }^{41}$ the viscosity of a disordered suspension can be written as

$$
\eta_{\text {iso }}=\frac{\eta_{0}-\eta_{\infty}}{1+\mathrm{Pe}}+\eta_{\infty}
$$

where $\eta_{\infty}$ and $\eta_{0}$ are the high- and low-frequency limiting viscosities, of expressions

$$
\begin{aligned}
& \eta_{\infty}=\beta\left(1-\frac{\Phi_{3 \text { iso }}}{0.63}\right)^{-2}, \\
& \eta_{0}=\beta\left(1-\frac{\Phi_{3 \text { iso }}}{0.71}\right)^{-2} .
\end{aligned}
$$

In Eq. (42), Pe is the Peclet number or the dimensionless angular frequency. This number measures the importance of hydrodynamic effects with respect to thermal ones. It is defined to be

$$
\mathrm{Pe}=\frac{6 \pi \beta R^{3} \mathcal{R}(\Omega)}{k_{B} T} .
$$

In the following, we shall assume that Eq. (41) still applies on condition to use expression (42) for the viscosity of the isotropic liquid.

The real part of the solution of Eq. (39) and its asymptotic limits given by Eqs. (40) and (41) are drawn in Fig. 4 for $\tau=0.1, \Phi_{3}=0.4$, and a radius of the colloidal par-

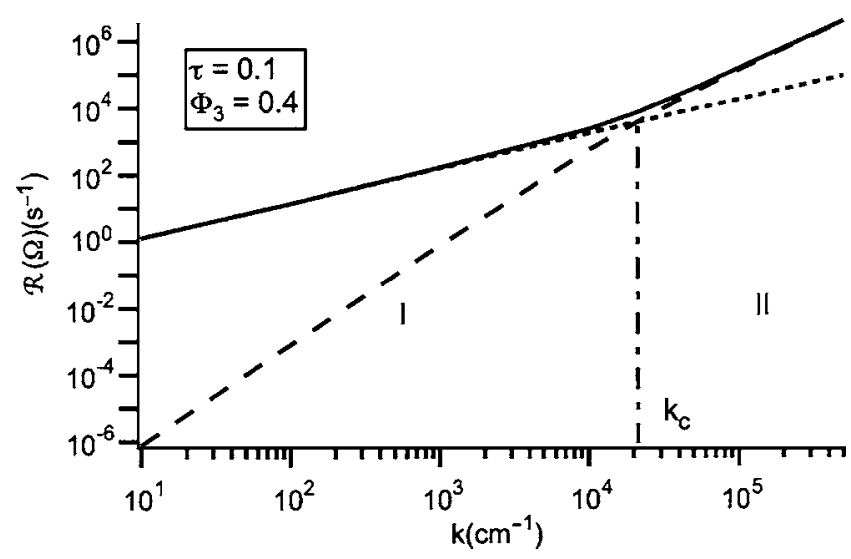

FIG. 4. The damping rate $\mathcal{R}(\Omega)$ as a function of $k$ for the colloid composition $\Phi_{3}=0.4$, reduced temperature $\tau=0.1$, and the fixed ratio $\Phi_{2} / \Phi_{1}$ $=0.25$. The general dispersion relation $[\mathrm{Eq}$. (39)] (continuous curve), the inner region dispersion relation [Eq. (40)] (dashed curve), and the outer region dispersion relation [Eq. (41)] (dotted curve). It must be noted that in the whole range of wave vectors shown in this graph, $\Omega$ is a real number so that $\mathcal{R}(\Omega)=\Omega$ (waves are overdamped).

ticles $R=100 \mathrm{~nm}$. To perform numerical calculations, we have taken the typical experimental values given in the literature for 5CB: $\alpha=\beta=0.01 \mathrm{~Pa} \mathrm{~s}$ and $\rho=10^{3} \mathrm{~kg} / \mathrm{m}^{3}{ }^{8}$. For the transport coefficient $\Gamma_{\Phi}$ introduced in Eq. (31), we have taken $\Gamma_{\Phi}=4.5 \times 10^{-13} \mathrm{~m}^{3} \mathrm{~s} / \mathrm{kg}$. This value has been estimated by assuming that the typical molecular velocities $v_{S}$ $=l_{S} / t_{S}$ and $v_{\Phi}=l_{\Phi} / t_{\Phi}$ are equal. Note that here $l_{S}$ $=\left(K_{S} S_{\text {nem } 0}^{2} / f_{0}\right)^{1 / 2}, t_{S}=3 \mu_{1} S_{\text {nem }}^{2} / 2 f_{0}, l_{\Phi}=\left(K_{\Phi} / f_{0}\right)^{1 / 2}$, and $t_{\Phi}$ $=K_{\Phi} / \Gamma_{\Phi} f_{0}^{2}$.

Two regions can be clearly distinguished. In the short wavelength limit (region II), the interface is diffuse and the relaxation of the two order parameters is the dominant process. The dispersion relation is given by Eq. (40) (dashed curve in Fig. 4). In the long wavelength limit, the viscous damping process in the outer region dominates and the corresponding dispersion relation is given by Eq. (41) (dotted curve in Fig. 4).

The transition between these two regions takes place when $\mathcal{R}\left(\Omega_{\eta}\right)=\mathcal{R}\left(\Omega_{S \Phi}\right)$. That gives the critical wave number $k_{c} \simeq 2.1 \times 10^{4} \mathrm{~cm}^{-1}$ corresponding to the critical wavelength $\lambda_{c} \simeq 3 \times 10^{-4} \mathrm{~cm}$. In Fig. 4, the influence of colloids on the dispersion relation is not clearly visible. For this reason, we superposed in Fig. 5 the curves representing the dispersion relations of a ternary and a binary mixture. More precisely, in Fig. 5, the solid line refers to the previous ternary mixture with $\Phi_{3}=0.4$ and $\Phi_{2} / \Phi_{1}=0.25$, whereas the dashed line has been calculated for a binary mixture with $\Phi_{2} / \Phi_{1}=0.25$ (and $\left.\Phi_{3}=0\right)$.

We observe that the presence of colloids tends to decrease the damping rate mainly in the hydrodynamic regime. The effect increases when the wavelength increases. This can be explained by considering the influence of two factors. First, the colloids decrease the nematic-isotropic interfacial tension (see Fig. 2). In the case of the ternary mixture with $\Phi_{3}=0.4$, the interfacial tension has the value $\gamma / \gamma_{S} \approx 4$, while for the binary mixture without colloids, $\gamma / \gamma_{S} \approx 21$. This effect tends clearly to decrease the damping rate. Second, the colloids increase the viscosity of the isotropic phase [see 


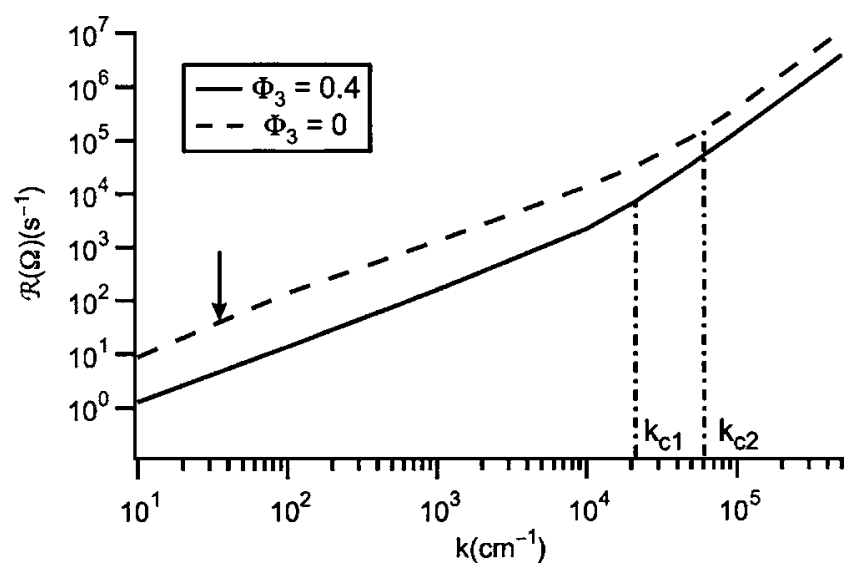

FIG. 5. The damping rate $\mathcal{R}(\Omega)$ as a function of $k$ for $\tau=0.1$ without colloids (dotted curve) and for a colloid concentration $\Phi_{3}=0.4$ (continuous curve). In both cases, $\Phi_{2} / \Phi_{1}=0.25$. Without colloids, waves are overdamped above $k \approx 33.6 \mathrm{~cm}^{-1}$ and start to propagate below. The arrow indicates the limit between these two regimes. In contrast, waves are overdamped in the case of $\Phi_{3}=0.4$ in the whole range of wave vectors shown in the figure (they would start to propagate below $k \approx 1.5 \mathrm{~cm}^{-1}$ ).

Eqs. (42) and (43)]. This effect also tends to decrease the damping rate. As can be seen from Fig. 6 where the viscosity of the isotropic phase [given by Eq. (42)] is plotted as a function of the wave vector for $\Phi_{3}=0.4$ and $\Phi_{2} / \Phi_{1}=0.25$, this effect is stronger below a typical wave vector of about $2 \times 10^{3} \mathrm{~cm}^{-1}$. More precisely, this curve shows that the colloids increase the viscosity by a factor of 7.5 (with respect to the value without colloids) below this limit and by a factor of 5.3 above. The sum of these two effects explains the increase by one order of magnitude of the damping rate observed at large wavelength (Fig. 5).

Coming back to Fig. 5, we shall note in addition that the value of $k_{c}$ that defines the "transition" between hydrodynamic and relaxation of the order parameter regimes differ in the two cases. Without colloids $k_{c}=k_{c 2} \approx 4.3 \times 10^{4} \mathrm{~cm}^{-1}$, while $k_{c}=k_{c 1} \approx 1.3 \times 10^{4} \mathrm{~cm}^{-1}$ in the presence of colloids. This result is expected inasmuch as the colloids tend to decrease the damping rate mainly in the hydrodynamic regime.

Finally, we shall note that our analysis only makes sense if the wavelength of the perturbation is larger than the mean distance between the colloidal particles. For colloidal particles of radius $R=100 \mathrm{~nm}$ and a concentration $\Phi_{3}=0.4$, this

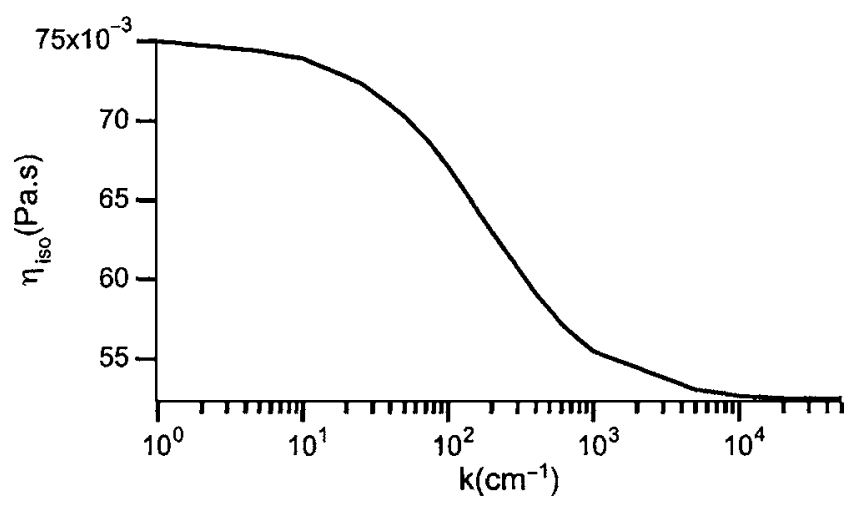

FIG. 6. The viscosity of the isotropic phase as a function of $k$ for $\tau=0.1$, for colloid concentration $\Phi_{3}=0.4$, and the ratio $\Phi_{2} / \Phi_{1}=0.25$. condition is satisfied as long as $k \leqslant\left(3 \Phi_{3} / 4 \pi\right)^{1 / 3}(2 \pi / R)$ $\approx 3 \times 10^{5} \mathrm{~cm}^{-1}$, which is the case in all our calculations.

\section{CONCLUSIONS}

In this paper we have examined a few properties of a three-component system composed of a liquid crystal, a nonnematogenic fluid (impurities), and colloids. This theoretical study was motivated by recent experimental observations showing that during the preparation of mixtures of a liquid crystal with PMMA spheres, a third component, alkane, slowly liberates from the particles and diffuses into the suspension. This phenomenon was shown to influence the mesh size of the cellular structure formed after the nematicisotropic phase separation, as well as its rheological properties.

In this context we have more explicitly studied the phase diagram and the static and dynamical properties of the nematic-isotropic interface. Our main results can be summarized as follows.

(1) If one adds colloids to a liquid crystal-impurity mixture of a given composition $\Phi_{2} / \Phi_{1}$, the colloids go into the isotropic phase (there are expelled from the nematic phase). In this case, we have shown that the impurity concentration decreases in the isotropic liquid so that the nematic-isotropic surface tension also decreases.

(2) Inversely, if one adds impurities to a liquid crystalcolloid mixture of fixed composition $\Phi_{1} / \Phi_{3}$, they concentrate into the rich-in-colloid isotropic liquid and the nematic-isotropic surface tension increases.

(3) Finally, capillary waves are strongly influenced by impurities and colloids. This comes from the fact that their dispersion relation strongly depends both on the viscosity of the two phases and on the surface tension. In particular, we have shown that adding colloids to a liquid crystal-impurity mixture of a given composition $\Phi_{2} / \Phi_{1}$ tends to decrease the damping rate of the waves because the surface tension decreases and the viscosity of the isotropic liquid increases.

We must nevertheless mention that our calculations are simplified as they neglect the coupling between the nematic director and the hydrodynamic flow, as well as the anchoring effect of the director at the interface. In addition, we did not use the complete set of Leslie viscosities and we assumed that the viscosity of the isotropic phase (without the colloids) is independent of the impurity concentration. In spite of these simplifications, we hope that our model retains the essential physics and will be helpful to better understand the microstructure and the rheological properties of the cellular networks formed in the coexistence region.

Finally, note that our calculations could be generalized in order to take into account the coupling between the interface oscillations, the director field, and the velocity. One could also include density effects, in particular, to study interfaces in lyotropic liquid crystals. 


\section{ACKNOWLEDGMENTS}

One of the authors (V.P.-N.) thanks Ecole Normale Supérieure de Lyon for scientific hospitality and acknowledges support from CEEX-05-D11-76 (SIDISANIZ) Research Program.

${ }^{1}$ E. M. Terentjev, Phys. Rev. E 51, 1330 (1995).

${ }^{2}$ V. A. Raghunathan, P. Richetti, and D. Roux, Langmuir 12, 3789 (1996).

${ }^{3}$ O. V. Kuksenok, R. W. Ruhwandl, S. V. Shiyanovskii, and E. M. Terentjev, Phys. Rev. E 54, 5198 (1996).

${ }^{4}$ R. W. Ruhwandl and E. M. Terentjev, Phys. Rev. E 55, 2958 (1997); 56, 5561 (1997)

${ }^{5}$ T. C. Lubensky, D. Pettey, N. Currier, and H. Stark, Phys. Rev. E 57, 610 (1998).

${ }^{6}$ H. Stark, Phys. Rep. 351, 387 (2001).

${ }^{7}$ P. G. de Gennes and J. Prost, The Physics of Liquid Crystals, 2nd ed. (Oxford University Press, New York, 1993).

${ }^{8}$ P. Oswald and P. Pieranski, Nematic and Cholesteric Liquid Crystals: Concepts and Physical Properties Illustrated by Experiments, Liquid Crystals Book Series (Taylor \& Francis, London/CRC, Boca Raton, 2005).

${ }^{9}$ P. Poulin, V. A. Raghunathan, P. Richetti, and D. J. Roux, J. Phys. III 4, 1557 (1994).

${ }^{10}$ P. Poulin, H. Stark, T. C. Lubenski, and D. A. Weitz, Science 275, 1770 (1997); P. Poulin and D. A. Weitz, Phys. Rev. E 57, 626 (1998).

${ }^{11}$ J.-C. Loudet, P. Barois, and P. Poulin, Nature (London) 407, 611 (2000).

${ }^{12}$ S. P. Meeker, W. C. K. Poon, J. Crain, and E. M. Terentjev, Phys. Rev. E 61, R6083 (2000).

${ }^{13}$ J. Yamamoto and H. Tanaka, Nature (London) 409, 321 (2001).

${ }^{14}$ V. J. Anderson, E. M. Terentjev, S. P. Meeker, J. Crain, and W. C. K. Poon, Eur. Phys. J. E 4, 11 (2001).

${ }^{15}$ V. J. Anderson and E. M. Terentjev, Eur. Phys. J. E 4, 21 (2001).

${ }^{16}$ J. Cleaver and W. C. K. Poon, J. Phys.: Condens. Matter 16, S1901 (2004).

${ }^{17}$ D. Vollmer, G. Hinze, W. C. K. Poon, J. Cleaver, and M. E. Cates, J. Phys.: Condens. Matter 16, L227 (2004).

${ }^{18}$ D. Vollmer, G. Hinze, B. Ullrich, W. C. K. Poon, M. E. Cates, and A. B.
Schofield, Langmuir 21, 4921 (2005).

${ }^{19}$ V. Popa-Nita, T. J. Sluckin, and S. Kralj, Phys. Rev. E 71, 061706 (2005).

${ }^{20}$ P. Ziherl, A. Šarlah, and S. Žumer, Phys. Rev. E 58, 602 (1998); A. Šarlah and S. Žumer, ibid. 60, 1821 (1999).

${ }^{21}$ P. van der Schoot, J. Phys. Chem. B 103, 8804 (1999).

${ }^{22}$ P. J. Flory, Principles of Polymer Chemistry (Cornell University Press, Ithaca, 1953).

${ }^{23}$ N. F. Carnahan and K. E. Starling, J. Chem. Phys. 51, 635 (1969).

${ }^{24}$ W. B. Russel, D. A. Saville, and W. R. Schowalter, Colloidal Dispersions (Cambridge University Press, Cambridge, 1989).

${ }^{25}$ S. Faetti and V. Palleschi, J. Chem. Phys. 81, 6254 (1984); Phys. Rev. A 30, 3241 (1984); J. Phys. (France) Lett. 45, L313 (1984).

${ }^{26}$ K. Denolf, B. Van Roie, C. Glorieux, and J. Thoen, Phys. Rev. Lett. 97, 107801 (2006).

${ }^{27}$ A. Matsuyama, R. M. L. Evans, and M. E. Cates, Eur. Phys. J. E 9, 79 (2002); Z. Lin, H. Zhang, and Y. Yang, Phys. Rev. E 58, 5867 (1998).

${ }^{28}$ R. Williams, Mol. Cryst. Liq. Cryst. 35, 349 (1976).

${ }^{29}$ S. Hess, Z. Naturforsch. A 31A, 1507 (1976).

${ }^{30}$ P. D. Olmsted and P. Goldbart, Phys. Rev. A 41, 4578 (1990); 46, 4966 (1992); P. D. Olmsted and C.-Y. D. Lu, Phys. Rev. E 60, 4397 (1999).

${ }^{31}$ T. Qian and P. Sheng, Phys. Rev. E 58, 7475 (1998).

${ }^{32}$ J. L. Ericksen, Arch. Ration. Mech. Anal. 4, 231 (1960).

${ }^{33}$ F. M. Leslie, Q. J. Mech. Appl. Math. 19, 357 (1966); Arch. Ration. Mech. Anal. 28, 265 (1968).

${ }^{34}$ J. W. Cahn, Trans. Metall. Soc. AIME 242, 166 (1968).

${ }^{35} \mathrm{M}$. H. Holmes, Introduction to Perturbation Methods (Springer-Verlag, New York, 1995)

${ }^{36}$ V. Popa-Nita and T. J. Sluckin, Phys. Rev. E 66, 041703 (2002).

${ }^{37}$ V. Popa-Nita and P. Oswald, Phys. Rev. E 68, 061707 (2003).

${ }^{38}$ V. Popa-Nita, P. Oswald, and T. J. Sluckin, Mol. Cryst. Liq. Cryst. 435, 215 (2005).

${ }^{39}$ L. D. Landau and E. M. Lifshitz, Fluid Mechanics (Pergamon, Oxford, 1959).

${ }^{40}$ V. G. Levich, Physicochemical Hydrodynamics (Prentice-Hall, Englewood Cliffs, NJ, 1962).

${ }^{41}$ C. G. de Kruif, E. M. F. van Iersel, and A. Vrij, J. Chem. Phys. 83, 4717 (1985). 